\title{
Tangent Dirac Structures and Poisson Dirac Submanifolds
}

\author{
Md. Showkat Ali*1 ${ }^{1}$, M. G. M Talukder ${ }^{2}$ and M. R. Khan ${ }^{2}$ \\ ${ }^{1}$ Department of Mathematics, Dhaka University, Dhaka-1000, Bangladesh \\ ${ }^{2}$ Department of Mathematics, Comilla University, Comilla, Bangladesh
}

(Received : 17 December 2012; Accepted : 31 July 2013)

\begin{abstract}
The local equations that characterize the submanifolds $N$ of a Dirac manifold $M$ is an isotropic (coisotropic) submanifold of $T M$ endowed with the tangent Dirac structure. In the Poisson case which is a result of $X u$ : the submanifold $N$ has a normal bundle which is a coisotropic submanifold of $T M$ with the tangent Poisson structure if and only if $N$ is a Dirac submanifold. In this paper we have proved a theorem in the general Poisson case that the fixed point set $M^{G}$ has a natural induced Poisson structure that implies a Poisson-Dirac submanifolds, where $G \times M \rightarrow M$ be a proper Poisson action.
\end{abstract}

Key words: Dirac structures, Poisson Dirac submanifolds, Poisson bracket.

\section{Introduction}

$X u^{1}$ has proved that the Dirac submanifolds of the Poisson manifold $(M, P)$ are characterized by the nice property of having a normal bundle which is a coisotropic submanifold of the tangent manifold $T M$ endowed with the tangent Poisson structure. All the terms of $X u$ 's result, including the notion of a tangent Dirac structure ${ }^{2}$, are also defined for Dirac manifolds, and $X u$ 's result indicates interesting connections between the geometry of a submanifold $N$ of a Dirac manifold $M$ and the geometry of a normal bundle of $N$ in the tangent manifold $T M$. This is the motivation ${ }^{1}$ of the present paper. We discuss the geometric configuration of $X u$ 's result in the general case of a Dirac manifold. The terms of the theorem are either new or not popular, and are based on either new or not popular geometric constructions. Accordingly, it is an objective of the paper to explain these terms in detail. Particularly, we recall the general construction of the vertical and complete lifts of tensor fields from a manifold $M$ to the total space of the tangent bundle $T M$, and the main properties of these operations ${ }^{3}$. We use these lifts in order to give a simple definition of the tangent Dirac structure and make some new remarks about it. Then, we turn to submanifolds. We define various classes of submanifolds of a Dirac manifold and characterize them via local coordinates and bases. Furthermore, we obtain the local conditions that characterize submanifolds of $(M, D)$ with a normal bundle $N$ which is either a coisotropic or an isotropic submanifold of $T M$. These formulas imply the result proved by $X u$ in the case of Poisson manifolds. Another consequence of the established formulas is that the analogs of Dirac submanifolds of a presymplectic manifold $M$ are characterized by the existence of a normal bundle which is isotropic in $T M$.

Theorem 1.1. Let $G \times M \rightarrow M$ be a proper Poisson action. Then the fixed point set $M^{G}$ has a natural induced Poisson structure.

Proper Poisson actions have been studied intensively in the last 15 years. For example, the theory of reduction for Hamiltonian systems has been developed extensively for these kinds of actions. We refer the reader to the recent monograph by Ortega and Ratiu ${ }^{4}$ for a survey of results in this area. Theorem 1.1 should have important applications in symmetry reduction and this is one of our main motivations for this work.

This paper is organized as follows: (i) Section 2 provides a simple definition of the tangent Dirac structure; (ii) Section 3 recalls the notion of a Poisson-Dirac submanifold; (iii) some related results needed for the proof of Theorem 1.1 presented in Section 4 and (iv) deduction of some consequences and their applications presented in Section 5.

\section{Tangent Dirac Structures}

Now, we use the complete and vertical lifts in order to define the notion of a tangent Dirac structure first introduced by Courant ${ }^{2}$ and make some new remarks about it. The Dirac structures are defined as a class of subbundles of the vector bundle $E(M)=T M \oplus T^{*} M$. The bundle $E(M)$ has several interesting geometric objects. The first is the non degenerate metric of zero signature $g((X, \alpha),(Y, \beta))=\frac{1}{2}(\alpha(Y)+\beta(X))$

where $\mathrm{X}, \mathrm{Y}$ are tangent vectors and $\mathrm{Q}, \mathrm{\square}$ are tangent covectors at $x \in M$ The second $F \in \Gamma E n d(E(M))$. is given by

$F(X, \alpha)=(X,-\alpha)$,

which is a so-called para-Hermitian structure ${ }^{5}$. The third object is the non degenerate 2-form

$((X, \alpha),(Y, \beta))=g((X, \alpha), F(Y, \beta))=\frac{1}{2}((\alpha(Y)-$ $\beta(X))$.

Definition 2.1. A maximal $g$-isotropic subbundle $A \subseteq$ $E(M)$ is called an almost Dirac structure on $\mathrm{M}$.

The almost Dirac structure can be interpreted in terms of $T M$ alone. The pair $(A, \varpi)$, where $A$ is the generalized distribution defined as the natural projection of $A$ on $T M$ and, $\forall x \in M, \varpi_{x} \in \wedge^{2} A_{x}^{*}$ is the 2-form induced by $\omega$ of (2.3) $\left(\forall X, Y \in A_{x}\right.$, the value produced by (2.3) does not depend on the choice of $\alpha, \beta)$. Conversely, the pair $(A, \varpi)$ allows us to reconstruct $A$ as follows:

* Author for correspondence. email: msa317@yahoo.com 


$$
A=\left\{(X, \alpha) /\left.X \in A \& \alpha\right|_{A}=i(X) \varpi\right\} .
$$

The next important thing for the bundle $E(M)$ is the Courant bracket $^{6}$, which is the operation defined on $\Gamma E(M)$. by

$$
\begin{aligned}
& {[(X, \alpha),(Y, \beta)]=} \\
& \left([X, Y], L_{X} \beta-L_{Y} \alpha+d(\omega((X, \alpha),(Y, \beta)))\right) \\
& =\left([X, Y], i(X) d \beta-i(Y) d \alpha+\frac{1}{2} d(\beta(X)-\alpha(Y))\right)
\end{aligned}
$$

where $X, Y$ are vector fields and $\alpha, \beta$ are differential 1-forms on $M,[X, Y]$ is the usual Lie bracket and $L$ denotes the Lie derivative. The Courant bracket is skew-symmetric but satisfies a more complicated than the Jacobi identity.

Definition 2.2. An almost Dirac structure $D \subseteq E(M)$ is called a Dirac structure on $M$ if $\Gamma D$ is closed by Courant brackets.

$\mathrm{Xu}^{1}$ proved that the almost Dirac structure is Dirac if and only if the equivalent pair $(A, \varpi)$ satisfies the following conditions:

i) $\quad \mathrm{A}$ is a generalized foliation,

ii) the form $\varpi$ is closed along the leaves of $\mathrm{A}$.

This means that the leaves of A are presymplectic manifolds and are called the presymplectic leaves of $D$. If the leaves are symplectic then $D$ is equivalent to a Poisson structure. Namely, if $P$ is the corresponding Poisson bi-vector field, the Dirac structure is

$D_{P}=\left\{(i(\alpha) P, \alpha) / \alpha \in T^{*} M\right\}$.

If the leaves are the connected components of $M, D$ is a presymplectic structure on $M$ with the presymplectic form $\varpi$ such that

$D=D_{\varpi}=\{(X, i(X) \varpi) / X \in T M\}$.

\section{Poisson-Dirac Submanifolds}

Let $M$ be a Poisson manifold. For background in Poisson geometry we refer the reader to Vaisman's book ${ }^{7}$. We denote the Poisson bi-vector field by $\pi \in \chi^{2}(\mathrm{M})$ so that the Poisson bracket is given by:

$$
\{f, g\}=\pi(d f, d g), \forall f, g \in C^{\infty}(M) .
$$

Recall that a Poisson submanifold $N \subset M$ is a submanifold which has a Poisson bracket and for which the inclusion $i: N \rightarrow M$ is a Poisson map:

$$
\{f \circ i, g \circ i\}_{M}=\{f, g\}_{N} \circ i, \quad \forall f, g \in C^{\infty}(N) .
$$

Such Poisson submanifolds are, in a sense, extremely rare. In fact, they are collections of open subsets of symplectic leaves of $M$.

Example 3.1. Let $M$ be a symplectic manifold with symplectic form $\omega$. Recall that a symplectic submanifold is a submanifold $i: N \rightarrow M$ such that the restriction $i * \omega$ is a symplectic form on $N$. For every even dimension $0 \leq$ $2 i \leq \operatorname{dim} M$, there are symplectic submanifolds of dimension $2 i$. On the other hand, the only Poisson submanifolds are the open subsets of $M$.

Crainic and Fernandes ${ }^{8}$ introduce the following natural extension of the notion of a Poisson submanifold:

Definition 3.1. Let $M$ be a Poisson manifold. A submanifold $N \subset M$ is called a Poisson-Dirac submanifold if $N$ is a Poisson manifold such that:

(i) the symplectic foliation of $N$ is

$$
N \cap F=\{L \cap N: L \in F\} \text {, and }
$$

(ii) for every leaf $L \in F, L \cap N$ is a symplectic submanifold of $L$.

Note that if $(M,\{\cdot, \cdot\})$ is a Poisson manifold, then the symplectic foliation with the induced symplectic forms on the leaves, gives a smooth foliation with a smooth family of symplectic forms. Conversely, given a manifold $M$ with a foliation $F$ furnished with a smooth family of symplectic forms on the leaves, then we have a Poisson bracket on $M$ defined by the formula

$$
\{f, g\} \equiv X_{f(g)}
$$

for which the associated symplectic foliation is precisely $F$. Hence, a Poisson structure can be defined by specifying its symplectic foliation ${ }^{9}$. It follows that a submanifold $N$ of a Poisson manifold $M$ has at most one Poisson structure satisfying conditions (i) and (ii) above, and this Poisson structure is completely determined by the Poisson structure of $M$.

Example 3.2. Let $L$ be a symplectic leaf of a Poisson manifold, and $N \subset M$ a submanifold which is transverse to $L$ at some $x_{0}$ :

$$
T_{x_{0}} M=T_{x_{0}} L \oplus T_{x_{0}} N
$$

Then one can check that conditions (i) and (ii) in Definition 3.1 are satisfied in some open subset in $N$ containing $x_{0}$. In other words, if $N$ is small enough then it is a Poisson-Dirac submanifold. Sometimes one calls the Poisson structure on $N$ the transverse Poisson structure to $L$ at $x_{0}$, up to Poisson diffeomorphisms, this structure does not depend on the transversal $N$.

The two conditions in Definition 3.1 are not very practical to use. Let us give some alternative criteria to determine if a given submanifold is a Poisson Dirac submanifold.

Observe that condition (ii) in the definition means that the symplectic forms on a leaf $L \cap N$ are the pull-backs $i * \omega_{L}$, where $i: N \cap L \rightarrow L$ is the inclusion into a leaf and $\omega_{L} \in$ $\Omega^{2}(L)$ is the symplectic form. Denoting by \#: $T * M \rightarrow$ $T M$ the bundle map determined by the Poisson bivector field, we conclude that we must have

$$
T N \cap \#\left(T N_{0}\right)=\{0\},
$$

Since the left-hand side is the kernel of the pull-back $i * \omega_{L}$. If this condition holds, then at each point $x \in N$ we obtain a bivector $\pi_{N}(x) \in \wedge^{2} T_{x} N$ and one can prove in Crainic and Fernandes ${ }^{8}$. 
Proposition 3.1. Let $N$ be a submanifold of a Poisson manifold $M$ such that

(a) equation (3.1) holds, and

(b) the induced tensor $\pi_{N}$ is smooth.

Then $\pi_{N}$ is a Poisson tensor and $N$ is a Poisson-Dirac submanifold.

Remark 3.1. Equation (3.1) can be interpreted in terms of the Dirac theory of constraints. This is the reason for the use of the term "Poisson-Dirac submanifold". We refer the reader to Crainic and Fernandes ${ }^{8}$ for more explanations.

On the other hand, from Proposition 3.1, we deduce the following sufficient condition for a submanifold to be a Poisson-Dirac submanifold.

Corollary 3.1. Let $M$ be a Poisson manifold and $N \subset M$ a submanifold. Assume that there exists a subbundle $E \subset$ $T_{N} M$ such that:

$$
T_{N} M=T N \oplus E \text { and } \#\left(E^{0}\right) \subset T N .
$$

Then $N$ is a Poisson-Dirac submanifold.

Proof. Under the assumptions of the corollary, one has a decomposition

$$
\mathrm{Q}=\pi_{N}+\pi_{E}
$$

where $\pi_{N} \in \Gamma\left(\wedge^{2} T N\right)$ and $\pi_{E} \in \Gamma\left(\wedge^{2} \mathrm{E}\right)$ are both smooth bivector fields. On the other hand, one checks easily that (2.1) holds. By Proposition 3.1, we conclude that $N$ is a Poisson-Dirac submanifold.

There are Poisson-Dirac submanifolds which do not satisfy the conditions of this corollary. Also, the bundle $E$ may not be unique. For a detailed discussion and examples we refer to Crainic and Fernandes ${ }^{8}$. Under the assumptions of the corollary, the Poisson bracket on the Poisson-Dirac submanifold $N \subset M$ is quite simple to describe: Given two smooth functions $f, g \in C^{\infty}(N)$, to obtain their Poisson bracket we pick extensions $\tilde{f}, \tilde{g} \in C^{\infty}(M)$ such that $d_{x} \tilde{f}, d_{x} \tilde{g} \in E_{x}^{0}$. Then the Poisson bracket on $N$ is given by:

$$
\{f, g\}_{N}=\left.\{\tilde{f}, \tilde{g}\}\right|_{N}
$$

It is not hard to check that this formula does not depend on the choice of extensions.

\section{Fixed Point Sets of Proper Poisson Actions}

In this section we will give a proof of Theorem 1.1, which we restate now as follows:

Theorem 4.1. Let $G \times M \rightarrow M$ be a proper Poisson action. Then the fixed point set $M^{G}$ is a Poisson-Dirac submanifold.

Since the action is proper, the fixed point set $M^{G}$ is an embedded submanifold of $M$. Its connected components may have different dimensions, but our argument will be valid for each such component, so we will assume that $M^{G}$ is a connected submanifold. The proof will consist in showing that there exists a subbundle $E \subset T_{M^{G}} M$ satisfy the conditions of Corollary 3.1. First of all, given any action $G \times M \rightarrow M$ (proper or not) there exists a lifted action $G \times T M \rightarrow T M$. For proper actions we have the following basic property:

Proposition 4.1. If $G \times M \rightarrow M$ is a proper action then there exists a $G$-invariant metric on $T M$.

For a proof of this fact and other elementary properties of proper actions, we refer to Duistermaat and $\mathrm{Kolk}^{10}$. Explicitly, the $G$-invariance of the metric means that:

$$
\langle g . v, g . w\rangle_{g . p}=\langle u, w\rangle_{p} \quad \forall u, w \in T_{p} M
$$

where $g \in G$ and $p \in M$.

We fix, once and for all, a $G$-invariant metric $\langle$,$\rangle for our$ proper Poisson action $G \times M \rightarrow M$. Let us consider the subbundle $E \subset T_{M^{G}} M$ which is orthogonal to $T M^{G}$ :

$$
E=\left\{v \in T_{M^{G}} M:\langle u, v\rangle=0, \forall w \in T M^{G}\right\} .
$$

Lemma 4.1. $T_{M^{G}} M=T M^{G} \oplus E$ and $\#\left(E^{0}\right) \subset T M^{G}$.

Proof. Since $E=\left(T M^{G}\right)^{\perp}$, the decomposition $T_{M^{G}} M=$ $T M^{G}$ is obvious. Now for a proper action, we have $(T M)^{G}=T M^{G}$ so this decomposition can also be written as:

$$
T_{M^{G}} M=(T M)^{G} \oplus E
$$

On the other hand, we have the lifted cotangent action $G \times T * M \rightarrow T * M$, which is related to the lifted tangent action by

$$
g \cdot \xi(v)=\xi\left(g^{-1} \cdot v\right), \xi \in T * M, v \in T M .
$$

We claim that

$$
E^{0} \subset(T * M)^{G} .
$$

In fact, if $v \in T M$ we can use (4.1) to decompose it as $v=v_{G}+v_{E}$, where $v_{G} \in(T M)^{M}$ and $v_{E} \in E$. Hence, for $\xi \in E^{0}$ we find

$$
\begin{aligned}
& g \cdot \xi=\xi\left(g^{-1} \cdot v_{G}+g^{-1} \cdot v_{E}\right) \\
& =\xi\left(v_{G}\right)+\xi\left(g^{-1} \cdot v_{E}\right) \\
& =\xi\left(v_{G}\right) \\
& \left.=\xi\left(v_{G}\right)+\xi\left(v_{E}\right)=\nabla_{G}+v_{E}\right) .
\end{aligned}
$$

We conclude that $g \cdot \xi=\xi$ and (4.2) follows.

Since $G \times M \rightarrow M$ is a Poisson action, we see that \# : $T * M \rightarrow T M$ is a $G$-equivariant bundle map. Hence, if $\xi \in E^{0}$, we obtain from (4.2) that:

$$
g \cdot \# \xi=\#(g \cdot \xi)=\# \xi .
$$

This means that $\# \xi \in(T M)^{G}=T M^{G}$,

so the Lemma holds.

This Lemma shows that the conditions of Corollary 3.1 are satisfied, so $M^{G}$ is a Poisson-Dirac submanifold and the proof of Theorem 4.1 is completed. 


\section{Applications}

Example 5.1. Let $\mathbb{C}^{n+1}$ be the complex $(n+1)$-dimensional space with holomorphic coordinates $\left(z_{0}, \ldots, z_{n}\right)$ and antiholomorphic coordinates $\left(\widetilde{z_{0}}, \ldots, \widetilde{z_{n}}\right)$. On the (real) manifold $\mathbb{C}^{n+1}-0$ we will consider a (real) quadratic Poisson bracket of the form:

$$
\left\{z_{i}, z_{j}\right\}=a_{i j} z_{i} z_{j},\left\{z_{i}, \widetilde{z_{j}}\right\}=\left\{\widetilde{z_{i}}, \widetilde{z_{j}}\right\}=0 \text {. }
$$

where $A=\left(a_{i j}\right)$ is a skew-symmetric matrix.

The group $\mathbb{C}^{*}$ of non-zero complex numbers acts on $\mathbb{C}^{n+1}-0$ by multiplication of complex numbers. This is a free and proper Poisson action, so the quotient $\mathbb{C} P(n)=$ $\mathbb{C}^{n+1}-0 / \mathbb{C}^{*}$ inherits a Poisson bracket.

Let us consider now the action of the $n$-torus $T^{n}$ on $\mathbb{C}^{n+1}$ - 0defined by:

$$
\left(\theta_{1}, \ldots, \theta_{n}\right) \cdot\left(z_{0}, z_{1}, \ldots, z_{n}\right)=\left(z_{0}, e^{i \theta_{1}} z_{1}, \ldots ., e^{i \theta_{n}} z_{n}\right) .
$$

This is a Poisson action that commutes with the $C^{*}$-action. It follows that the $T^{n}$-action descends to a Poisson action on $\mathbb{C} P(n)$. Note that the action of $T^{n}$ on $\mathbb{C} P(n)$ is proper but not free. The quotient $\mathbb{C} P(n) / T^{n}$ is not a manifold but it can be identified with the standard simplex

$$
\Delta^{n}=\left\{\left(\mu_{0}, \cdot \cdot \cdot \mu_{n}\right) \in R^{n+1}: \sum_{i=0}^{n} \mu_{i}=1, \mu_{i} \geq 0\right\} .
$$

This identification is obtained via the map $\mu: \mathbb{C} P(n) \rightarrow$ $\Delta^{n}$ defined by:

$$
\begin{aligned}
& \mu\left(\left[z_{0}, z_{1}, \cdot \cdot z_{n}\right]\right) \\
& =\left(\frac{\left|z_{0}\right|^{2}}{\left|z_{0}\right|^{2}+\cdot \cdot \cdot\left|z_{n}\right|^{2}} \cdot \cdot \cdot \frac{\left|z_{n}\right|^{2}}{\left|z_{0}\right|^{2}+\cdot \cdot \cdot\left|z_{n}\right|^{2}}\right)
\end{aligned}
$$

Let us describe the Poisson stratification of $\Delta^{n}=\mathbb{C} P(n) / T^{n}$. The Poisson bracket on $\Delta^{n}$ is obtained through the identification

$$
C^{\infty}\left(\Delta^{n}\right) \simeq C^{\infty}\left(\mathbb{C} P(n) / T^{n}\right.
$$

For that, we simply compute the Poisson bracket between the components of the map

straightforward computation will show that

$$
\begin{aligned}
& \left\{\mu_{i}, \mu_{j}\right\}=\left(a_{i j}-\sum_{l=0}^{n}\left(a_{i l}+a_{l j}\right) \mu_{l}\right) \mu_{i} \mu_{j} \\
& i, j=0, \cdot \cdot n .
\end{aligned}
$$

Now notice that (5.1) actually defines a Poisson bracket on $\mathbb{R}^{n+1}$. For this Poisson bracket, the interior of the simplex and its faces are Poisson submanifolds: a face $\Delta_{i_{1} \ldots, i_{n-d}}$ of dimension $0 \leq d \leq n$ is given by equations of the form:

$$
\begin{aligned}
\sum_{i=0}^{n} \mu_{i}=1, \mu_{i_{1}}= & , \ldots, \mu_{i_{n-d}}=0, \mu_{i}>0 \text { for } i \neq \\
& \left\{i_{1}, \ldots, i_{n-d}\right\}
\end{aligned}
$$

These equations define Poisson submanifolds since

(a) the bracket $\left\{\mu_{i}, \mu_{l}\right\}$ vanishes whenever $\mu_{l}=0$, and

(b) the bracket $\left\{\mu_{i} \sum_{l=0}^{n} \mu_{l}\right\}$ vanishes whenever $\sum_{l=0}^{n} \mu_{l}=1$.

Therefore, the Poisson stratification of $\Delta^{n}$ consists of strata formed by the faces of dimension $0 \leq d \leq n$, which are smooth Poisson manifolds.

Example 5.2. Let $M$ be a Poisson manifold and $N \subset M$ a submanifold. Assume that there exists a subbundle $E \subset$ $T_{N} M$ such that $E^{0}$ is a Lie subalgebroid of $T *$ $M$ (equivalently, $E$ is a co-isotropic submanifold of the tangent Poisson manifold $T M$ ). Then $E$ satisfies the assumptions of the corollary 3.1, so $N$ is a Poisson-Dirac submanifold. This class of Poisson-Dirac submanifolds has very special geometric properties. They were first studied by $X u^{1}$ which calls them Dirac submanifolds. They are further discussed by Crainic and Fernandes ${ }^{8}$, where they are called Lie-Dirac submanifolds.

\section{References}

1. Xu, P., 2003. Dirac submanifolds and Poisson involutions. Ann. Sci. Ec. Norm. Sup., 36, 403 - 430

2. Courant, T. J., 1990. Tangent Dirac Structures. J. Phys. A, 23, 5153 - 5168.

3. Yano, K and S. Ishihara, 1973. Tangent and Cotangent Bundles. M. Dekker, Inc., New York.

4. Ortega, J.-P., Ortega and T. Ratiu, 2004. Momentum maps and Hamiltonian reduction. Progress in Mathematics, 222, Birkhäuser, Boston.

5. Cruceanu, V., P. Fortuny and P. M. Gadea, 1996. A survey on paracomplex geometry. Rocky Mountain J. of Math., 26, 1-33.

6. Courant, T. J., 1990. Dirac Manifolds. Transaction Amer. Math. Soc., 319, 631- 661.

7. Vaisman, I., 1994. Lectures on the Geometry of Poisson

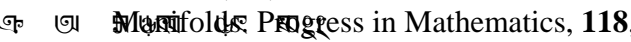

Birkhäuser, Berlin.

8. Crainic, M. and R. L., Fernandes, 2004. Integrability of Poisson brackets. Journal of Differential Geometry 66, 71137.

9. Vaisman, I., 2005. Foliation coupling Dirac structures arXiv:math.SG/0412318

10. Duistermaat, J. and J., Kolk, 2000. Lie Groups. SpringerVerlag Berlin Heidelberg. 
Dhaka Univ. J. Sci. 62(1): 21-24, 2014 (January)

*Author for correspondence.email: msa317@yahoo.com 